\title{
A Modified Method for Speckle Noise Removal in Ultrasound Medical Images
}

\author{
T.Ratha Jeyalakshmi and K.Ramar
}

\begin{abstract}
Ultrasound images contain speckle noise which degrades the quality of the images. Eliminating such noise is an important preprocessing task. This paper describes and analyses an algorithm for cleaning speckle noise in ultrasound medical images. Mathematical Morphological operations are used in this algorithm. This algorithm is based on Morphological Image Cleaning algorithm (MIC) designed by Richard Alan Peters II. The algorithm uses a different technique for reconstructing the features that are lost while removing the noise. For morphological operations it also uses arbitrary structuring elements suitable for the ultrasound images which have speckle noise.
\end{abstract}

Index Terms-bottom hat, morphology, reconstruction, speckle, structuring element, top hat

\section{INTRODUCTION}

Ultrasound imaging is widely used in the field of medicine. It is used for imaging soft tissues in organs like liver, kidney, spleen, uterus, heart, brain etc. The speed, low cost of imaging and the portability of scanning machine makes it very popular. The common problem in Ultrasound image is speckle noise which is caused by the imaging technique used that may be based on coherent waves such as acoustic to laser imaging [8][9]. This paper describes and analyses an algorithm for reducing such speckle noise. This algorithm is based on mathematical morphology. It is a modified form of MIC and it is called as MMIC. It differs from MIC by not using the histogram for calculating the threshold of the image. It is also using a different technique for reconstructing the features that are of speckle's size. Moreover it uses structuring elements which are having arbitrary structures which resemble the shapes of the speckles. This algorithm produces better result when compared to the original MIC in time complexity as well as output quality. This paper is organized as follows. Section II discusses the previous works in the literature. Section III describes the MIC and the modifications done to that in this paper. Section IV gives the modified version of MIC. The results and discussion are given in Section V.

\section{RELATED WORKS}

Various techniques for speckle noise removal are available

T.Ratha Jeyalakshmi is a Research scholar at Mother Teresa University, Kodaikanal, Tamil Nadu, India. (radha_jeyalakshmi@yahoo.com)

Dr.K.Ramar is Professor and Head in Department of Computer Science and Engineering, National Engineering College, Kovilpatti, Tamil Nadu, India. (kramar_nec@rediffmail.com in the literature [11][13][14][17][19][25]. Linear filtering techniques like spatial averaging have blurring effect [4]. Adaptive filtering techniques based on local statistics [2],[9][20] or spectral coefficients [11] are good in preserving object boundaries and small features with speckle size. Application of non linear filters is also available in the literature. Non linear filters based on Mathematical morphology are size and shape sensitive and they are found to be good in removing speckle patterns [4][5][6][7]. Morphological filters use mathematical morphological operations such as opening, closing, top hat, bottom hat etc. [10][12][13][16]. A morphological algorithm known as Morphological Image Cleaning algorithm is good in reducing noise in different types of images including scanner images[3]. This algorithm finds the residual image which is the difference between the original image and the smoothed image. It separates the features from the residual image and puts it back into the original image so that features are preserved. This algorithm is an iterative procedure which works with disk shaped structuring elements with different radius. First it filters the original image repeatedly using Opening Closing and Closing opening (OCCO) filter using disk shaped structuring elements of different radius. The output of each iteration is processed as follows. The positive elements of the residue are put in an array and the other elements are put in a different array. For each array threshold is calculated using the second order moment of the gray level distribution shown by the histogram. Then each thresholded image is cleaned as follows. Rank order filter is applied and the isolated pixels are deleted repetitively until no more isolated pixels are found. Thresholding ends in trimming of bases of features. To recover those bases of the features MIC expands the nonzero regions by skeletonizing and dilating. The first cleaned thresholded image is added to and the second cleaned thresholded image is subtracted from the output of OCCO before continuing the next iteration which takes this result as the input.

\section{MODIFICATIONS MADE TO MIC}

Since the cleaning process takes much time the algorithm is modified such that it does less processing to get the result. First for filtering using OCCO the arbitrary structuring elements that resemble the shape of the speckle are used. A speckle does not have a regular shape [15]. It is not appropriate to use predefined structuring elements like disk, rectangle, hexagon etc., for morphological processing [9] [19]. Therefore an arbitrary structuring element which resembles the speckle shape is designed. For this random 
speckle samples are taken from different ultrasound images and by thresholding three structuring elements were designed as given in fig. 1. In MIC for thresholding the image, histogram of the image is used. In MMIC instead of that the standard deviation of the pixels in the image is used and from this the threshold is found. The morphological operations like opening and closing which are used for filtering in OCCO will lose the features which are less than or equal to the size of the structuring element. For reconstructing the features that are lost while cleaning, opening by reconstruction and closing by reconstruction are used [1][10] These are efficient techniques for getting back the lost image features.

$\begin{array}{ccc}001100 & 000000000000 & 00000000000000 \\ 011111 & 000111100000 & 00001111000000 \\ 000000 & 011111111110 & 00111111110000 \\ & 011111111100 & 0011111111110 \\ & & 0111111111110 \\ & & 0111111111110\end{array}$

Fig. 1 Arbitrary structuring elements a1, a2 and a3

Grayscale opening by reconstruction is defined as follows.

Let $\mathrm{I}$ and $\mathrm{J}$ be two grayscale images defined on the same domain $\mathrm{D}_{\mathrm{I}}$ such that $\mathrm{I}<=\mathrm{J}$. The grayscale reconstruction by opening is obtained by iterative grayscale geodesic erosions of $\mathbf{J}$ above I until stability is reached.

$$
\rho_{\mathrm{I}}(\mathrm{J})=\hat{\mathrm{n} \geq 1}_{\mathrm{I}}^{\varepsilon^{(\mathrm{n})}(\mathrm{J})}
$$

Grayscale closing by reconstruction is defined as follows.

Let $\mathrm{I}$ and $\mathrm{J}$ be two grayscale images defined on the same domain $\mathrm{D}_{\mathrm{I}}$ such that $\mathrm{I}<=\mathrm{J}$. The grayscale reconstruction by closing is obtained by iterative grayscale geodesic dilations of $\mathrm{J}$ under I until stability is reached.

$$
\rho_{\mathrm{I}}(\mathrm{J})=\underset{\mathrm{n} \geq 1}{\mathrm{~V}} \varepsilon_{\mathrm{I}}^{(\mathrm{n})}(\mathrm{J})
$$

\section{MMIC ALGORITHM}

Step1. Let $\mathrm{i}=0$

Step2. Let $\mathrm{i}=\mathrm{i}+1$

Step3. Let $X=\operatorname{OCCO}\left(I, a_{i}\right)$ where $I$ is the original image with noise and $\mathrm{a}_{\mathrm{i}}$ be the arbitrary structure element.

Step4. Let $\mathrm{T}(\mathrm{x}, \mathrm{y})=$ tophat $(\mathrm{I}, \mathrm{X})$

Step5. Find $\mathrm{t}(\mathrm{x}, \mathrm{y})$ by thresholding $\mathrm{T}(\mathrm{x}, \mathrm{y})$ using the standard deviation of $\mathrm{T}(\mathrm{x}, \mathrm{y})$

Step6. Let tcap= reconstruction by closing of $t$

Step7. Let $\mathrm{B}(\mathrm{x}, \mathrm{y})=$ bothat $(\mathrm{I}, \mathrm{X})$

Step8. Find $b(x, y)$ by thresholding $B(x, y)$ using the standard deviation of $\mathrm{B}(\mathrm{x}, \mathrm{y})$

Step9. Let bcap $=$ reconstruction by opening of $b$.

Step10. Modify I as X+tcap-bcap

Step11. Go to step 2 if $i<=3$.

\section{V. PARAMETERS USED FOR ANALYZING THE OUTPUT OF THE ALGORITHM}

The assessment parameters such as Noise Standard Deviation (NSD), Mean Square Error (MSE), Equivalent Numbers of Looks (ENL), Peak Signal to Noise Ratio(PSNR) and Execution time are used to asses the algorithm MMIC[17][22][24].

\section{A. Noise Standard Deviation (NSD)}

This determines the quantity of the speckle in the image. If the quantity of speckle is less the NSD will be less. The formula for NSD is

$$
\begin{gathered}
\text { NSD }=\text { SORT }\left(\left(\sum(\mathrm{DI}(\mathrm{x}, \mathrm{y})-\mathrm{NMV})^{2}\right) /\left(\mathrm{m}^{*} \mathrm{n}\right)\right. \\
\mathrm{x}=1, \mathrm{~m} \\
\mathrm{y}=1, \mathrm{n} \\
\mathrm{NMV}=(\Sigma(\mathrm{DI}(\mathrm{x}, \mathrm{y})) /(\mathrm{m} * \mathrm{n}) \\
\mathrm{x}=1, \mathrm{~m} \\
\mathrm{y}=1, \mathrm{n}
\end{gathered}
$$

Where DI is de noised image, $m$ represents the number of rows and $\mathrm{n}$ represents the number of columns in the image.

\section{B. Mean Square Error (MSE)}

The Mean Square Error is used to find the total amount of difference between two images. It indicates average difference of the pixels throughout the image where DI is the de noised image, and I is the original image with speckle noise. A lower MSE indicates a smaller difference between the original Image with speckle and de noised image. The formula is

$$
\begin{aligned}
& \operatorname{MSE}=\left(\Sigma(\mathrm{x}, \mathrm{y})-\mathrm{DI}(\mathrm{x}, \mathrm{y})^{2}\right) /(\mathrm{m} * \mathrm{n}) \\
& \mathrm{x}=1, \mathrm{~m} \\
& \mathrm{y}=1, \mathrm{n}
\end{aligned}
$$

\section{Equivalent Numbers of Looks (ENL)}

To estimate the speckle noise level another assessment parameter known as ENL over a uniform region is used. A larger value of ENL shows a better quantitative performance. The formula used to calculate ENL is

$$
\mathrm{ENL}=\mathrm{NMV}^{2} / \mathrm{NSD}^{2}
$$

\section{Peak Signal to Noise Ratio (PSNR)}

It is an assessment parameter to measure the performance of the speckle noise removal method. The formula is

$$
\mathrm{PSNR}=10 \log _{10}\left(255^{*} 255 /(\mathrm{MSE})\right)
$$

TABLE I. PERFORMANCE ANALYSIS OF MIC and MMIC WITH ULTRASOUND image of neonatal brain with size 144 X 109

\begin{tabular}{|c|c|c|c|c|c|}
\hline \multirow{2}{*}{$\begin{array}{c}\text { Algorith } \\
\mathbf{m}\end{array}$} & \multicolumn{5}{|c|}{ Assessment Parameters } \\
\cline { 2 - 6 } & NSD & MSE & ENL & PSNR & $\begin{array}{c}\text { Time } \\
\text { Elapsed } \\
\text { (secs) }\end{array}$ \\
\hline
\end{tabular}


International Journal of Computer and Electrical Engineering, Vol. 2, No. 1, February, 2010 1793-8163

\begin{tabular}{|c|c|c|c|c|c|}
\hline $\begin{array}{c}\text { MIC with } \\
\text { d1,d2,d3 }\end{array}$ & $\begin{array}{c}7.61136 \\
9 \mathrm{e}+001\end{array}$ & $\begin{array}{c}5.001 \\
4 \mathrm{e}+00 \\
3\end{array}$ & 4.6523 & 11.1399 & 4.07800 \\
\hline $\begin{array}{c}\text { MIC with } \\
\text { a1,a2,a3 }\end{array}$ & $\begin{array}{c}5.29591 \\
8 \mathrm{e}+001\end{array}$ & $\begin{array}{c}778.3 \\
0\end{array}$ & 1.9859 & 19.2193 & 2.89100 \\
\hline $\begin{array}{c}\text { MMIC } \\
\text { with } \\
\text { d1,d2,d3 }\end{array}$ & $\begin{array}{c}4.82411 \\
\mathrm{e}+001\end{array}$ & $\begin{array}{c}398.0 \\
0\end{array}$ & 1.3180 & 22.1320 & 1.61000 \\
\hline $\begin{array}{c}\text { MMIC1 } \\
\text { with } \\
\mathbf{a 1 , a 2 , a 3}\end{array}$ & $\begin{array}{c}5.24108 \\
8 \mathrm{e}+001\end{array}$ & $\begin{array}{c}775.1 \\
6\end{array}$ & 1.9976 & 19.2369 & 1.34400 \\
\hline
\end{tabular}

TABLE II. PERFORMANCE analysis of MIC and MMIC WITH ULTRASOUND image OF LIVER with size 305 X 290

\begin{tabular}{|c|c|c|c|c|c|}
\hline \multirow{2}{*}{ Algorithm } & \multicolumn{5}{|c|}{ Assessment Parameters } \\
\cline { 2 - 6 } & NSD & MSE & ENL & PSNR & $\begin{array}{c}\text { Time } \\
\text { Elapsed } \\
\text { (secs) }\end{array}$ \\
\hline $\begin{array}{c}\text { MIC with } \\
\text { d1,d2,d3 }\end{array}$ & $\begin{array}{c}7.7389 \\
33 \mathrm{e}+00 \\
1\end{array}$ & 790.6067 & 16.0048 & 19.1512 & 15.812 \\
\hline $\begin{array}{c}\text { MIC with } \\
\text { a1,a2,a3 }\end{array}$ & $\begin{array}{c}7.7792 \\
71 \mathrm{e}+00 \\
1\end{array}$ & 736.7259 & 13.6294 & 19.4128 & 13.735 \\
\hline $\begin{array}{c}\text { MMIC } \\
\text { with } \\
\text { d1,d2,d3 }\end{array}$ & $\begin{array}{c}7.9751 \\
41+00 \\
1\end{array}$ & 2.3444 & 6.6412 & 4.4305 & 6.7650 \\
\hline $\begin{array}{c}\text { MMIC1 } \\
\text { with } \\
\text { a1,a2,a3 }\end{array}$ & $\begin{array}{c}7.7758 \\
04 \mathrm{e}+00 \\
1\end{array}$ & 751.6463 & 13.6960 & 19.3707 & 4.2030 \\
\hline
\end{tabular}



(a)



(c)



(b)



(d)

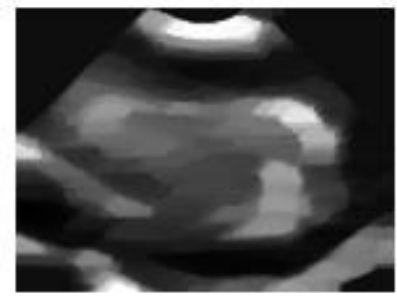

(e)

Fig. 2 a) ultra sound image of a neonatal brain with speckle noise b) MIC with 3 disk structures c) MIC with 3 arbitrary structures d) MMIC with 3 disk structures e) MMIC with 3 arbitrary structures

TABLE III. PERFORMANCE analysis of MIC and MMIC with ultrasound image of appendix with size 295 X 271

\begin{tabular}{|l|l|l|l|l|l|}
\hline \multirow{2}{*}{$\begin{array}{l}\text { Algorith } \\
\text { m }\end{array}$} & NSD & MSE & ENL & PSNR & Time \\
\cline { 2 - 6 } & $\begin{array}{c}\text { Elapsed } \\
\text { (secs) }\end{array}$ \\
\hline $\begin{array}{l}\text { MIC } \\
\text { with } \\
\text { d1,d2,d3 }\end{array}$ & $\begin{array}{c}5.1103 \\
1\end{array}$ & $3.7000 \mathrm{e}+00$ & 31.0034 & 12.4487 & 17.922 \\
\hline $\begin{array}{l}\text { MIC } \\
\text { with } \\
\text { a1,a2,a3 }\end{array}$ & $\begin{array}{l}4.6996 \\
1\end{array}$ & $\begin{array}{l}1.0269 \mathrm{e}+00 \\
\text { (1) }\end{array}$ & 11.3937 & 18.0153 & 14.828 \\
\hline $\begin{array}{l}\text { MMIC } \\
\text { with } \\
\text { d1,d2,d3 }\end{array}$ & $\begin{array}{l}4.5956 \\
12 \mathrm{e}+00\end{array}$ & 0.8827 & 4.7590 & 48.6728 & 6.1880 \\
\hline $\begin{array}{l}\text { MMIC1 } \\
\text { with } \\
\text { a1,a2,a3 }\end{array}$ & $\begin{array}{l}4.7454 \\
63 \mathrm{e}+00\end{array}$ & $1.0488 \mathrm{e}+00$ & 11.5934 & 17.9239 & 3.8120 \\
\hline
\end{tabular}



(a)



(c)



(b)



(d)



(e)

Fig 3 a) Ultra sound image of appendix with speckle noise b) MIC with 3 disk structures c) MIC with 3 arbitrary structures d) MMIC with 3 disk structures e) MMIC with 3 arbitrary structures 


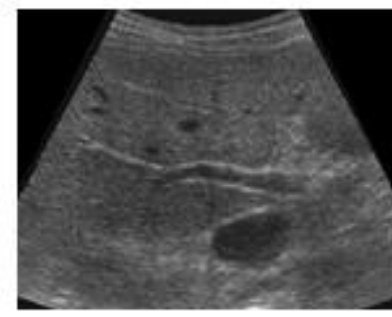

(a)

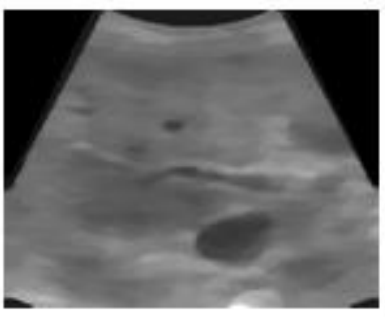

(c)



(b)



(d)

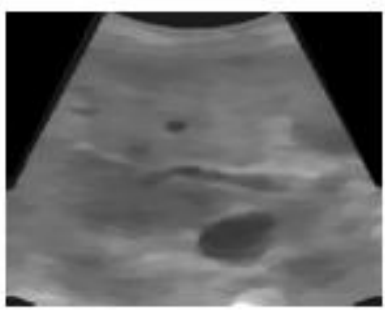

(e)

Fig 4 a) ultra sound image of liver with speckle noise b) MIC with 3 disk structures c) MIC with 3 arbitrary structures d) MMIC with 3 disk structures e) MMIC with 3 arbitrary structures



(a)



(c)

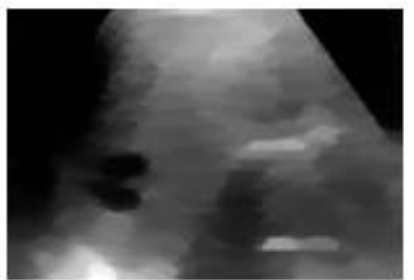

(b)



(d)
Fig. 5 Images cleaned by MMIC and segmented which shows uterine fibroid and liver cyst.

\section{RESULTS AND DISCUSSION}

The existing MIC algorithm has been modified as mentioned earlier in the paper. The Algorithm MMIC has been applied on different ultrasound images with different sizes. The output of the algorithm with their respective original input images are shown in Fig. 2, 3, and 4. The tables 1, 2 and 3 show the result of the algorithm on the three different ultrasound images. The images that are used in this paper are an image of neonatal brain, an image of appendix, and an image of liver. The tables show values of the assessment parameters such as NSD, MSE, ENL, PSNR, and Execution time for the MIC algorithm with three disk structures (of radius 3, 5, 9), for the MMIC with three disk structures (of radius 3, 5, 9) and for the MMIC with three arbitrary structures that resemble the speckle in the image. MMIC with a1, a2, a3 takes least execution time. MMIC with $\mathrm{d} 1, \mathrm{~d} 2, \mathrm{~d} 3$ looks the best when MSE is considered. MMIC with a1, a2, a3 is the best when all the parameters are considered. The subjective appearance is also good for the output of MMIC algorithm. Fig. 5 shows the images which have been cleaned by MMIC and further segmented. This proves that MMIC is the best algorithm for cleaning speckle noise which also preserves features in the image. Thus the image cleaned by this algorithm could be used as an input for other image processing tasks such as segmentation, feature extraction, classification etc.

\section{REFERENCES}

[1] Luc Vincent, "Morphological grayscale reconstruction in image analysis applications and efficient algorithms", IEEE Transactions in Image Processing, Vol.2, No.2, pp. 176-201, April 1993.

[2] M.Karamam, M. Kutay and G.Bozdagi, "An adaptive speckle suppression filter for medical ultrasonic images", IEEE Transactions on Medical Imaging Vol. 14, No.2, pp. 283-292,1995

[3] Richard Alan Peters II, “A new algorithm for image noise reduction using mathematical morphology", IEEE Transactions on Image Processing Vol.4, No.3, pp. 554-568,1995

[4] Mark A.Schulze and Qing X. Wu, "Noise Reduction in synthetic aperture radar imagery using a morphology-based nonlinear filter", Proceedings of DICTA95,pp. 661-666, 1995

[5] Salembier and J.Serra, "Flat zones filtering, connected operators and filters by reconstruction”, IEEE Transactions on Image Processing, Vol. 4, No. 8, August 1995

[6] Petros Marsgos, "Differential Morphology and Image Processing", IEEE Transaction on Image Processing, Vol. 5, No.6, June 1996.

[7] H.Heijmans, "Self dual morphological operators and filters", Journal of Mathematical Imaging and vision vol. 6, No.1 pp. 15-36, 1996

[8] L.Gagnon and A. Jouan, " Speckle filtering of SAR images - A comparative study between complex-wavelet-based and standard filters", SPIE proc. \#3169, 1997

[9] B.Aiazzi, L. Alparone and S.Baronti, "Multiresolution local statistics speckle filtering based on ratio laplacian pyramid", IEEE Transaction on Geoscience and Remote Sensing Vol. 36, No. 5, 1998

[10] Alla Vichik, Renato Keshet and David Malah1, "Self-dual morphology on tree semilattices and applications", Proceedings of the $8^{\text {th }}$ International Symposium on Mathematical Morphology, Rio de aneiro, Brazil, MCT/INPE, vo1. 1, pp. 49-60, 2007.

[11] L.Yaroslavsky and B.Zshaick "Transform oriented image processing technology for quantitative analysis of fetal movements in ultrasound image sequences", European Signal Processing Conference pp. 1745 1748, 1998

[12] P.Soillel, "Morphological image analysis", Springer _ Verly Berlin, 1999

[13] Holger Lange, "Advanced gray-scale morphological filters for the detection of sea mine in side scan sonar imagery", Proc. SPIE Vol. 4038, pp. 362-372, August 2000

[14] Volker Metzler, Marc Plus and Til Aach, "Restoration of ultrasound images by nonlinear scale space filtering", Institute of Signal Processing Medical University of Lubec, Germany, 2000

[15] Gjenna Stippel, Nilifred Philips Ignace Lemahieu, "A new denoising technique for ultrasound images using morphological properties of speckle combined with tissue classifying parameters", SPIE, Medical Imaging Conference Proceedings, No. 4687, p.p. 324-333, 2002

[16] Alan Yuille, Luc Vincent Geigery, " Statistical morphology and Bayesian reconstruction", Journal of Mathematical Imaging and Vision, Vol. 1, No. 3, August 1992

[17] Mastriani, A. E. Giraldez, "Smoothing of coefficients in wavelet domain for speckle reduction in Synthetic Aperture Radar images", ICGST International Journal on Graphics, Vision and Image Processing, Special Issue on Denoising, 2007

[18] BUADES, B. COLL,AND J. M. MOREL, “ A review of image denoising algorithms, with a new one", Multiscale Model. Simul. Vol. 4, No. 2, pp. $490-530,2005$ 
[19] Yael Erez, Yoav Y. Schechner, and Dan Adam, "Ultrasound image denoising by spatially varying frequency compounding", Proc. DAGM Symposium, Lecture notes on computer science Vol. 4147, pp. 1-10, 2006

[20] Vladimir Melnik, " Nonlinear locally adaptive techniques for image Filtering and restoration in mixed noise environments", Thesis for the degree of Doctor of Technology Tampere University of Technology, 2000

[21] K.Sivakami Sundari, V. Sadasivam, "Reduction of artifacts in JPEG images with genetic algorithm and boundary", GVIP Special Issue on Denoising, 2007

[22] C.A. Glasbey, M. Berman, H.M. Hudson, Encyclopaedia of image Biostatistics: analysis and tomography", 1998

[23] S.M.D. Mansoor Roomy, IM. Lakshmi V. Abhai Kumar, "A recursive gaussian weighted filter for impulse noise removal", GVIP Special Issue on Denoising, 2007

[24] Kazuhiro Hoshino, Frank Nielsen, Toshihiro Nishimura, "Noise reduction in cmos image sensors for high quality imaging: the autocorrelation function filter on burst image sequences", GVIP Special Issue on Denoising, 2007

[25] Hadeel Nasrat Abdullah, Mohammed Fadhil Hasan and Quassy Salim Tawfeeq, "Speckle noise Reduction in SAR Images Using double density dual tree DWT", Asian Journal of Information Technology Vol. 7, No.7,pp.281-284, 2008

T. Ratha Jeyalakshmi received M.C.A degree from Thiagarajar College of Engineering, Tamil Nadu, India and M.Phil degree from Manonmaniam Sundaranar University, Tamil Nadu, India. Currently she is pursuing Ph.D in computer Science at Mother Teresa University, Tamil Nadu, India. Her current research interests include pattern recognition and image processing.

K. Ramar is the Professor and Head of Computer Science and Engineering Department in National Engineering College, Kovilpatti, Tamil Nadu, India.

He received B.E. degree from Government College of Engineering, Tirunelveli, Tamil Nadu, India, and M.E. degree from PSG College of Technology, Tamil Nadu, India. He received Ph.D. degree from Manonmaniam Sundaranar University, Tamil Nadu, India. His area of interest includes Fuzzy Neural Network, Computer Vision System, Pattern Recognition, Data mining and Signal Processing 